ARTICLE

\title{
Human papillomavirus (HPV) vaccination of adolescents in the South African private health sector: Lessons from the HPV demonstration project in KwaZullu-Natal
}

\author{
N Tathiah, ${ }^{1}$ BSc (Hons), MB ChB, Dip HIV Man, MS (Epi), MMed (PHM), FCPHM; \\ M Naidoo, ${ }^{2}$ MB ChB, MFamMed, FCFP, MSc (Sports Medicine), Dip HIV Man, DipPEC, PhD; I Moodley, ${ }^{3} \mathrm{PhD}$ \\ ${ }^{1}$ Inkosi Albert Luthuli Central Hospital and Discipline of Public Health Medicine, College of Health Sciences, University of KwaZulu-Natal, \\ Durban, South Africa \\ ${ }^{2}$ Discipline of Family Medicine, School of Nursing and Public Health, College of Health Sciences, University of KwaZulu-Natal, Durban, \\ South Africa \\ ${ }^{3}$ Discipline of Public Health Medicine, School of Nursing and Public Health, College of Health Sciences, University of KwaZulu-Natal, Durban, \\ South Africa
}

Corresponding author: N Tathiah (tathiah@ukzn.ac.za)

In South Africa (SA), >4 000 women die annually of cervical cancer, a disease caused by the human papillomavirus (HPV). Infections caused by certain genotypes of HPV increase the risk of cervical cancer. HIV-infected women in particular are more likely to have persistent HPV infection, with higher-risk genotypes. In SA, two vaccines (HPV quadrivalent (types 6, 11, 16, and 18) vaccine, recombinant (Gardasil) and HPV bivalent (types 16 and 18) vaccine, recombinant (Cervarix)) are currently registered for the prevention of HPV-related disease. In the past, there have been significant challenges to achieving high coverage and uptake of vaccination - contributory factors include cost and lack of awareness. An HPV demonstration project among schoolgirls in rural KwaZulu-Natal showed that high vaccine uptake is achievable. In 2014, the National Department of Health launched the national HPV vaccination programme among female learners attending public schools. Awareness of HPV vaccination among healthcare providers, education of parents, teachers and learners, and avoidance of missed opportunities for vaccination are vital to the success of the programme. Primary healthcare practitioners may play an important role in cervical cancer prevention by identifying and offering vaccination to girls who miss the opportunity to be vaccinated at school. HPV vaccination should be considered as one arm of a comprehensive programme of cervical cancer prevention and control.

S Afr Med J 2015;105(11):954. DOI:10.7196/SAMJ.2015.v105il1.10135

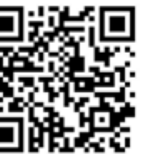

In South Africa (SA), cervical cancer is diagnosed in $>7700$ women each year, with $>4000$ women dying annually of the disease. ${ }^{[1]}$ Cervical cancer is caused by human papillomavirus (HPV) infection, the most common viral infection of the reproductive tract. ${ }^{[2]}$ More than 190 types of HPV have been identified; these are classified as high- or low-risk groups, depending on their potential to cause cancerous lesions. ${ }^{[2]}$

HPV infection results in a number of conditions in males and females, ranging from precancerous lesions to those that become cancerous. ${ }^{[2]}$ While the majority of HPV infections may result in spontaneous resolution without symptoms or disease, persistent infection with high-risk types may result in disease. ${ }^{[2]}$ In women, persistent infection with HPV high-risk types 16 and 18 may lead to precancerous lesions, which if left untreated may progress to cervical cancer. For males and females, $90 \%$ of all cases of anogenital warts are caused by HPV high-risk types 6 and $11 .{ }^{[2]}$ HIV-positive women have a higher prevalence and persistence of HPV infections, with an increased progression to cervical cancer. ${ }^{[2]}$

Cervical HPV infection is diagnosed using tests based on HPVDNA performed on cervical or vaginal swabs. Routine screening is done by cytology or visual inspection of the cervix by staining with acetic acid. HPV-induced changes in cervical epithelium are detected by the Papanicolaou (Pap) test. Precancerous lesions are treated by cryotherapy or surgical excision of the affected areas. ${ }^{[2]}$

In SA, there are currently two vaccines registered for the prevention of HPV-related disease: a quadrivalent vaccine (against HPV types 6,
11, 16 and 18 (Gardasil)) and a bivalent vaccine (against HPV types 16 and 18 (Cervarix)). ${ }^{[2]}$ Both vaccines are intended for prophylactic use before the onset of sexual activity and exposure to HPV. ${ }^{[2]}$

The quadrivalent vaccine is indicated for use in females and males $\geq 9$ years of age. ${ }^{[2]}$ It can be administered according to a 3 -dose $(0,2$, 6 months) or 2-dose (0 and 6 months) schedule. The second dose should be given a minimum of 1 month after the first dose, and the third dose a minimum of 3 months after the second dose. ${ }^{[2]}$ The bivalent vaccine is indicated for use in males and females $\geq 9$ years of age. The recommended dosing schedule is 0 and 6 months. The second dose can be given between 5 and 7 months after the first dose. ${ }^{[2]}$ The need for booster doses for either the quadrivalent or bivalent vaccines has not been established. Both vaccines should be administered intramuscularly $(0.5 \mathrm{~mL})$ in the deltoid region. ${ }^{[2]}$ The vaccines should be stored in a refrigerator $\left(2-8^{\circ} \mathrm{C}\right)$ and should not be frozen. ${ }^{[2]}$ Neither vaccine contains live biological products and are non-infectious; they also do not contain any preservative agents. ${ }^{[2]}$ Studies have shown that both vaccines are safe and highly immunogenic after administration. Current evidence indicates that the 2-dose schedule in females ( 9 - 14 years) is non-inferior to the 3 -dose schedule. ${ }^{[2]}$ The vaccines have been shown to be safe and immunogenic - also in HIV-positive individuals. ${ }^{[2]}$

Countries such as the USA, Australia, the UK, Canada and France offer HPV vaccination as part of a national immunisation programme ${ }^{[3]}$ In SA, historically, there has been a limited uptake of the HPV vaccine in the private sector, where estimates indicate that 
approximately 50000 individuals may have been vaccinated with the HPV vaccine between 2009 and 2014. ${ }^{[4]}$ Contributory factors may have been the high cost of the vaccines (approximately ZAR650 per dose of vaccine), and a paucity of knowledge and awareness around cervical cancer and HPV, and vaccine availability among healthcare workers and patients. ${ }^{[4]}$

\section{HPV demonstration project in KwaZulu-Natal}

An HPV demonstration project was conducted among 963 female learners ( $9-12$ years) in rural KwaZulu-Natal (KZN) and showed a high uptake of the vaccine, using the delivery model of school health teams. The uptake of the vaccine was $99.7 \%, 97.9 \%$ and $97.8 \%$ for the first, second and third doses, respectively. ${ }^{[5]}$

The high level of HPV vaccine delivery and uptake seen in this demonstration project is most likely owing to a combination of factors. Firstly, there was intensive communication with and education of all stakeholders, including the Department of Health $(\mathrm{DOH})$ and the Department of Education (DOE) at national, provincial and district level, school governing bodies, parents, learners, educators, healthcare workers, community leaders and the media prior to initiating vaccination. Community engagement and mobilisation was an integral part of this process, as was the role of the media in promoting awareness. Secondly, there was emphasis on obtaining informed consent from parents or guardians in a timely manner so that questions and concerns with regard to the safety and other long-term effects could be satisfactorily addressed. The third factor involved the optimisation of aspects of the healthcare system considered crucial to the success of the vaccination project. These included the leadership of members of the DOH and DOE, the project team, management of the human resource capacity (including training of the existing school health teams), and partnerships that existed between different government departments and the community, and between public and the private sectors. Other key aspects of the healthcare system included information management, which was important in terms of monitoring delivery, uptake and adverse events, and management of the cold chain and infrastructure. ${ }^{[5]}$

The high vaccine uptake observed in this HPV demonstration project is comparable to findings from other studies. ${ }^{[6-8]}$ A schoolbased HPV vaccination programme in Brazil also found high rates of vaccine uptake $-87.5 \%, 86.3 \%$ and $85.0 \%$ for the three doses, respectively. ${ }^{[6]} \mathrm{A}$ multicountry study of vaccination programmes in Peru, Uganda, Vietnam and India showed a high coverage in schoolbased programmes in the first 3 countries $(>80 \%) \cdot{ }^{[7]}$ In India, where a set of mixed strategies were used (school-based healthcare centres and community campaigns), coverage varied between $68.4 \%$ and $87.8 \%$, depending on the populations targeted. ${ }^{[7]}$

A study of the vaccination of 87000 girls ( 9 - 18 years) in 7 lowincome countries (Bhutan, Bolivia, Cambodia, Cameroon, Haiti, Lesotho and Nepal) using school-based strategies, health facility approaches, and combination school and facility models found a range of coverage rates depending on the delivery model. ${ }^{[8]}$ Overall, for all the countries, the model using the health facility alone had a coverage of $77 \%$, while the school-based and combination strategies had a coverage $>90 \% .{ }^{[8]}$ While school-based programmes have generally exhibited high uptake or coverage rates, the opposite has been shown in non-school-based programmes.

In the USA, which uses a non-school-based model, coverage has been reported as $35 \%$ for all 3 doses. ${ }^{[9]} \mathrm{HPV}$ vaccination was introduced into the routine immunisation schedule in 2006 for girls 11 or 12 years old, with a recommendation for catch-up doses in females aged $13-26$ years. ${ }^{[10]}$ In 2011, routine vaccination of males in the same age groups as the female target population was recommended. The vaccine type HPV prevalence decreased by $>50 \%$ (from 11.5\% to 5.1\%). ${ }^{[10]}$ The US Centers for Disease Control and Prevention (CDC) highlighted three areas to be addressed to improve the HPV vaccination coverage: the education of parents, as many do not understand the need for the vaccine or have concerns over its safety; healthcare providers who will be involved in recommending the vaccine - some are less likely to recommend the HPV vaccine than they would other vaccines; and the need to reduce missed opportunities for vaccination during healthcare visits. ${ }^{[9]}$

Experiences from HPV vaccine demonstration projects in other countries have shown that public messaging around cervical cancer, HPV, and the HPV vaccine has an important impact on the acceptability and effectiveness of vaccination programmes. ${ }^{[6-8]}$ The success of the vaccination programme in Brazil is attributed to the school-based advertising strategy and the role of the media. ${ }^{[6]}$ The information disseminated in schools played a significant role in the parents consenting to vaccinate their children. Also, the learners had an important part in actively conveying information from schools to their homes. ${ }^{[6-8]}$

A report of a school-based HPV vaccination programme in Peru highlighted the feasibility of using the existing resources in the healthcare system. ${ }^{[11]}$ Key elements related to the preparedness of the healthcare system were human resources, training, cold chain and infrastructure, and the recording and reporting of vaccinations. ${ }^{[1]}$ The facilitating factors were planning, co-ordination between education and health, facilitation between levels of the healthcare system, training, and comprehensive community awareness strategies. ${ }^{[1]}$

\section{DOH roll-out of HPV vaccines in schools}

In April 2014, the SA DOH launched the national school-based HPV vaccination programme for Grade 4 ( $\geq 9$ years old) female learners attending public schools, with a target population of an estimated 500000 learners. ${ }^{[12]}$ A 2-dose strategy was adopted, using the bivalent vaccine (Cervarix) ${ }^{[12]}$ The vaccination programme has been included in the Extended Programme on Immunisation (EPI). ${ }^{[12]}$ Prior to the commencement of the vaccination process, the Department of Basic Education (DBE) released a Guide for Educators, and made available vaccination consent forms, letters to parents and caregivers, and promotional material. ${ }^{[12]}$ Unpublished preliminary data indicate that the coverage is $>90 \%$ for both the first and second rounds of vaccination. ${ }^{[4]}$

\section{Implications for the primary healthcare provider}

Despite the HPV vaccine being rolled out through the school health teams to female learners in Grade 4 ( $\geq 9$ years), it is anticipated that some learners may miss the opportunity to be vaccinated because the vaccine is offered to public school learners only. Other learners may miss vaccination owing to absenteeism or no access to school health teams. ${ }^{[13]}$ This affords the primary healthcare practitioner the opportunity to contribute towards primary prevention of cervical cancer. Essential to this is the ability to identify girls who are eligible for the HPV vaccination school programme but have missed the opportunity, and to provide appropriate counselling to their parents. A clinical algorithm (Fig. 1) is outlined, which may be of use to the practising general practitioner or primary healthcare provider.

The Children's Act 38 of 2005 provides for a child $\geq 12$ years of age with sufficient emotional and mental maturity, who is able to comprehend the risks and benefits of treatment, and can consent to medical treatment without consent from a parent or guardian. ${ }^{[14]}$ The 


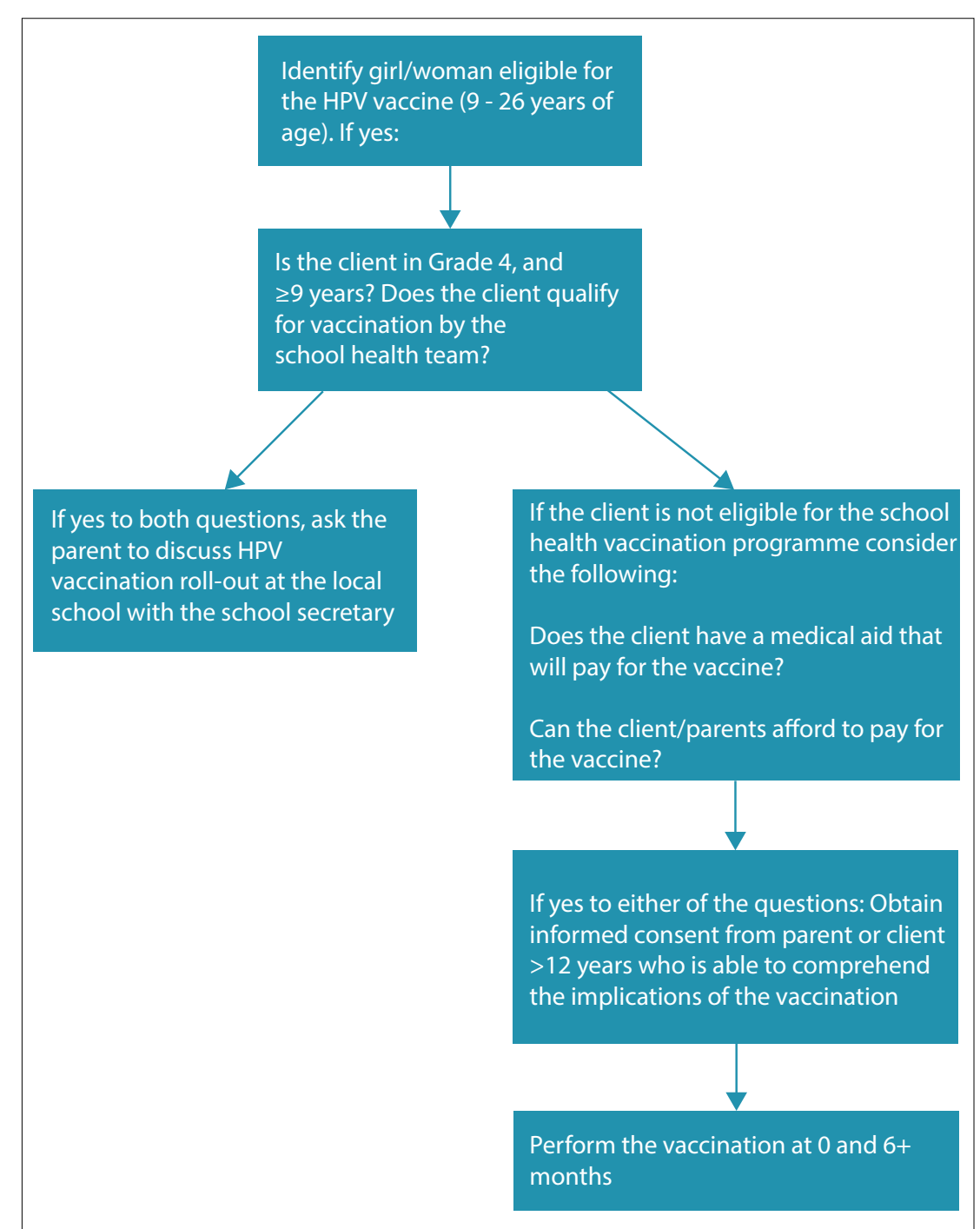

Fig. 1. Clinical algorithm for healthcare providers.

implication is that a child $\geq 12$ years meeting these criteria can independently consent to HPV vaccination. However, children $<12$ years require parental consent; the DBE provides information and forms for parents to sign before the visit by school health teams.

\section{Case vignette}

A 9-year-old girl is asked to get her parents to sign consent forms for the administration of the HPV vaccine. The forms are signed by the mother and the child is administered the vaccine 2 days later when the school health team visits the school. Subsequently, the child develops a fever for a few days, which resolves spontaneously. The parents are upset and approach the school for an explanation. The parents state that they were not informed of this possible side-effect of the vaccine.
Learning point: It is important to note that signing the consent form may not necessarily imply informed consent; therefore, it is important that members of the public are given the opportunity to ask questions about the intervention. The Health Professions Council of South Africa (HPCSA) states that the legal requirements for proper informed consent in SA should include the following:

- 'Knowledge of the nature or extent of the harm or risk;

- Appreciated and understood the nature of the harm or risk;

- Consented to the harm or assumed the risk; and

- The consent must have been comprehensive (i.e. extended to the entire transaction, inclusive of its consequences). ${ }^{[15]}$
Recommendations

\section{for private healthcare} providers to increase awareness and improve uptake of HPV vaccination among adolescents

In view of the demonstrated success of vaccinating learners in a school-based HPV vaccination delivery model, it is recommended that the private sector increase knowledge and awareness around cervical cancer, HPV and the need for HPV vaccination, especially in adolescents. This strategy should be viewed as part of a country-wide comprehensive programme for cervical cancer prevention and control that targets girls and women across each stage of their life course.

\section{References}

1. Bruni L, Barrionuevo-Rosas L, Serrano B, et al. Institut Català d'Oncologia (ICO) Information Centre on HPV and Cance (HPV Information Centre). Human papillomavirus and related diseases in South Africa. Summary Report. 2014. www. hpvcentre.net (accessed 26 March 2014).

2. World Health Organization. Human papillomavirus vaccines WHO position paper. Weekly Epidemiological Record 2014;43(89):465-492.

3. Boyce T, Holmes A. Addressing health inequalities in the delivery of the human papillomavirus vaccination programme: Examining the role of the school nurse. PLoS One 2012;7(9):e3416. [http:// dx.doi.org/10.1371/journal.pone.0043416]

4. Richter K. Implementation of HPV Vaccination in South Africa. 4. Richter K. Implementation of HPV Vaccination in South
Pretoria: Public Health Association of South Africa, 2015.

5. Moodley I, Tathiah N, Mubaiwa V, et al. High uptake of Gardasil vaccine among 9 - 12-year-old schoolgirls participating in an HPV vaccination demonstration project in KwaZulu-Natal, South Africa. S Afr Med J 2013;103(5):318-321. [http://dx.doi. org/10.7196/samj.6414]

6. Fregnani JHTG, Carvalho AL, Eluf-Neto J, et al. A school-based human papillomavirus vaccination program in Barretos, Brazil: Final results of a demonstrative study. PloS One 2013;8(4):e62647. [http://dx.doi.org/10.1371/journal.pone.0062647]

7. LaMontagne DS, Barge S, Nga TL, et al. Human papillomavirus vaccine delivery strategies that achieved high coverage in low- and middle-income countries. Bull world Health Organ 2011;89:821-830B. [http://dx.doi.org/10.2471/ BLT.11.08986]

8. Ladner J, Besson M, Hampshire R, et al. Assessment of eight HPV vaccination programs implemented in lowest income countries. BMC Public Health 2012;12:370. [http://dx.doi org/10.1186/1471-2458-12-370]

9. Centers for Disease Control and Prevention (CDC). Human papillomavirus vaccination coverage among adolescent girls, 2007-2012, and post licensure vaccine safety monitoring, 20062013. MMWR Morb Mortal Wkly Rep 2013:591.

10. Markowitz LE, Hariri S, Lin C, et al. Reduction in human papillomavirus (HPV) prevalence among young women following HPV vaccine introduction in the United States, National Health and Nutrition Examination Surveys, 2003-2010. I Infect Dis 2013;208(3):385-393. [http://dx.doi.org/10.1093/ infdis/jit192]

11. Penny M, Bartolini R, Mosqueira NR, et al. Strategies to vaccinate against cancer of the cervix: Feasibility of a school-based HPV vaccination program in Peru. Vaccine 2011;29(31):5022-5030 vaccination program in Peru. Vaccine $2011 ; 29(31) .50$
[http://dx.doi.org/10.1016/j.vaccine.2011.04.078]

12. National Department of Health. Human papilloma virus (HPV) vaccination campaign. 2014. http://www.health.gov.za/
(Hational Department of Health. Human papilloma virus (accessed 26 March 2014)

13. Botha M, Richter K. Cervical cancer prevention in South Africa: HPV vaccination and screening both essential to achieve and maintain a reduction in incidence. S Afr Med J 2015;105(1):33 35. [http://dx.doi.org/10.7196/samj.9233]

14. McQuoid-Mason DJ. Can children aged 12 years or more refuse life-saving treatment without consent or assistance from anyone else? S Afr Med J 2014;104(7):466-467. [http://dx.doi. org/10.7196/samj.8417]

15. Health Professions Council of South Africa (HPCSA). HPCSA Guidelines for Good Practice in the Health Care Professions: Seeking Patients' Informed Consent: The Ethical Considerations. Seeking Patients' Informed Consent: The 